\title{
Atypical localization of demodicosis after COVID-19 infection.
}

\author{
Ines Lahouel $^{1}$, Randa Said El Mabrouk ${ }^{1}$, Rim Hadhri ${ }^{1}$, Monia Youssef ${ }^{1}$, Hichem
}

Belhadjali ${ }^{1}$, and Jameleddine Zili ${ }^{1}$

${ }^{1}$ Fattouma Bourguiba University Hospital of Monastir

December 31, 2021

\begin{abstract}
Since its outbreak in December 2019, a consistent number of case reports have been published describing a complex spectrum of skin manifestations associated with COVID-19. We report a first observation of demodicidosis of the scalp after a severe acute respiratory syndrome coronavirus 2 (SARS-COV-2) infection.
\end{abstract}

Title: Atypical localization of demodicosis after COVID-19 infection.

Running title : Scalp demodicosis after COVID-19

The category for which the article is being submitted : Clinical image

Authors:

Ines Lahouel MD (1) (2): Medical doctor of dermatology

Randa Said El Mabrouk MD (1) (2): Medical doctor of dermatology

Rim Hadhri MD (3): Medical doctor of anatomopathology

Monia Youssef MD PhD (1) (2): Clinical professor of dermatology,

Hichem Belhadjali MDPhD (1) (2): Clinical professor of dermatology,

Jameleddin Zili MD Ph D (1) (2): Clinical professor of dermatology,

\section{Affiliations:}

(1) Dermatology department, Fattouma Bourguiba Hospital, University of Medicine, Monastir, Tunisia.

(2) Dermo-respiratory research laboratory, the University of Medicine of Monastir, Tunisia.

(3) Anatomo-pathology department, University of Medicine, Monastir, Tunisia.

Contributors' Statement Page:

Ines Lahouel: Writing the manuscript.

Randa Said El Mabrouk: Writing the manuscript and submitting the revised article.

Rim Hadhri: Wiriting the histological part of the manuscript.

Monia Youssef: Supervised and approved the revised manuscript.

Hichem Belhadjali: Supervised and approved the revised manuscript

Jameleddin Zili: Supervised and approved the revised manuscript 
Consent statement: approved by all authors

Written informed consent was also obtained from the patient to publish this report in accordance with the journal' patient consent policy"

Ethical statement : approved

Statement of acknowledgement : none

We choose this statement of Data availability : Data openly available in a public repository that issues datasets with DOIs

\section{Corresponding author:}

Randa Said El Mabrouk, MD,

Department of Dermatology, Fattouma Bourguiba Hospital, 5000 Monastir, Tunisia

Email: saidranda@yahoo.fr Phone: +216 54492241

Key words: demodicosis, scalp, COVID 19, immunodepression, dermatology, parasitology.

Word counts for the text: 193

Source of support: none

Acknowledgments: none

Conflict of interest: none

\section{Case presentation :}

A 45-year-old male patient presented with a lesion of the scalp appearing 10 days after a SARS-COV-2 infection. The skin examination found many firm and erythematous papules on a background of a diffuse erythema associated with hair thinning. Skin biopsy revealed excessive Demodex mites in the follicular infundibulum with perivascular and perifollicular lymphocytic infiltration. Based on the clinical and histopathological data, the diagnosis of scalp demodecidosis was retained. The patient was treated with topical metronidazole, achieving a complete regression of the lesions.

\section{Discussion:}

COVID-19 caused a variety of dermatological manifestations. Demodicidosis is an ectoparasitosis caused by the proliferation of a mite: Demodex $s p$, that permanently resides in or near the pilosebaceous unit and seborrheic glands. It has been implicated as a pathogen in innumerable skin conditions and typically in blepharitis and facial dermatitis such as rosacea-like or papulo-pustular eruptions ${ }^{1}$. The symptoms of scalp demodicidosis are not common, and can be found mainly in people with weakened immune systems ${ }^{2}$. Immunodeficiency appears to create a favourable environment for the development of the parasite.

In our case, disturbances of the host's immune response caused by SARS-COV-2 explains this atypical localization of demodicidosis.

\section{Key clinical message :}

Our observation emphasizes the importance of recognizing the cutaneous manifestations of COVID-19 given their variability and the necessity of a careful and critical skin evaluation in this pandemic period.

\section{References:}

1. Fernandez-Flores A, Alija A. Scalp folliculitis with Demodex: innocent observer or pathogen? Braz J Infect Dis. $2009 ; 13(2): 81-2$.

2. Seyhan ME, Karincaoğlu Y, Bayram N, Aycan O, Kuku I. Density of Demodex folliculorum in hematological malignancies. J Int Med Res. 2004;32(4):411-5. 


\section{Figures legends:}

Figure: 1: Diffuse erythema with erythematous papules associated with hair thinning in the occipital scalp.

Figure 2a: Lymphocytic infiltrate around follicles (Hematoxylin Eosin X 100).

Figure 2b: Multiple Demodex mites within pilosebaceous follicle (Hematoxylin Eosin X 400).
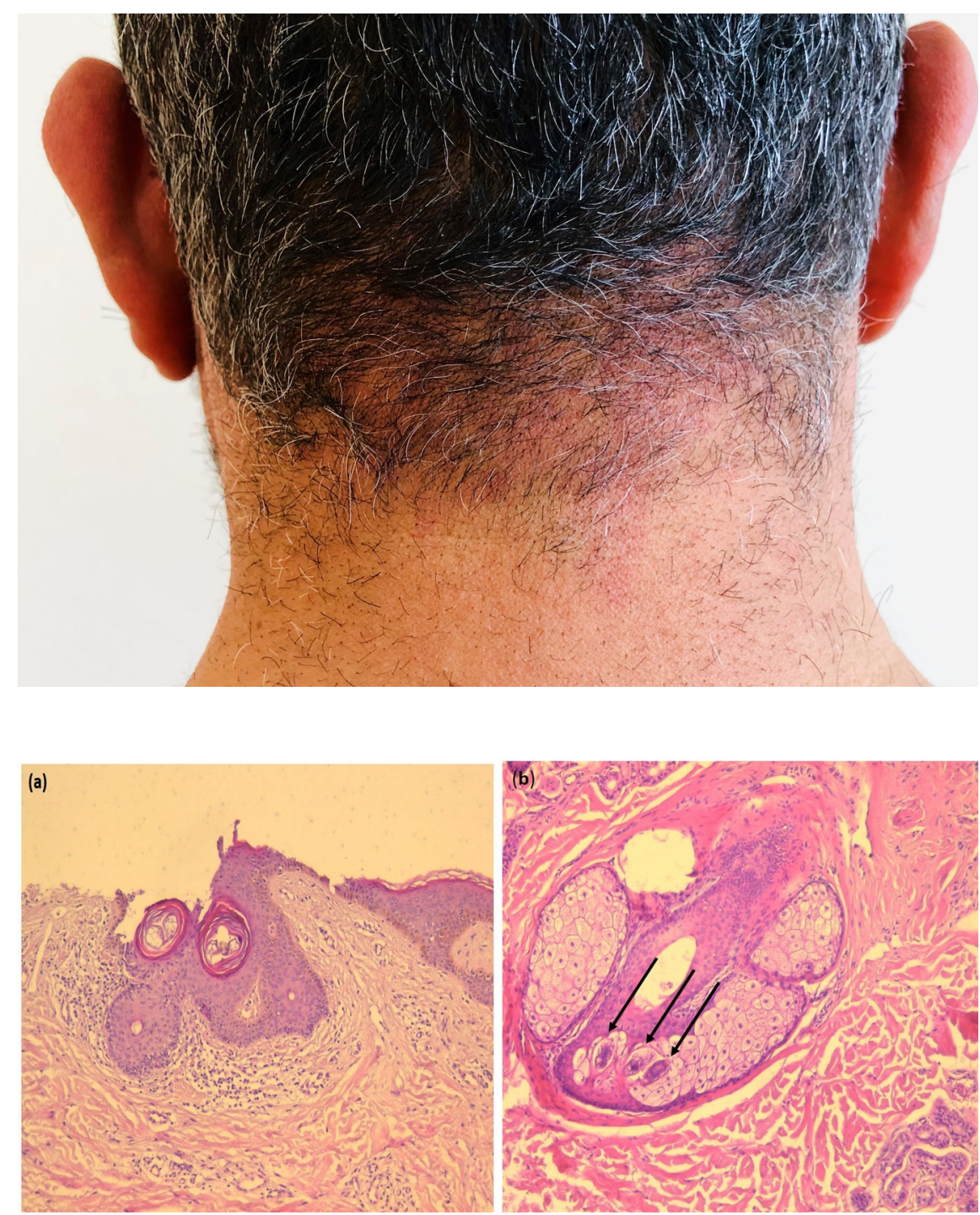